\title{
Recent trends in diagnosis of urogenital tuberculosis
}

\author{
Promod K Mehta*,1 \& Ekta Kamra ${ }^{1}$ \\ ${ }^{1}$ Centre for Biotechnology, Maharshi Dayanand University, Rohtak 124001, India \\ *Author for correspondence: Tel.: +91 9896504 193; Fax: +91 1262274 640; pkmehta3@hotmail.com
}

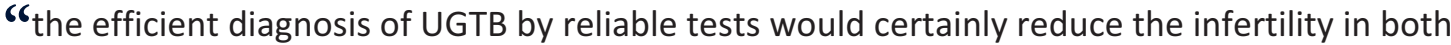
men and women and the other sequelae associated with disease."
\end{abstract}

First draft submitted: 22 November 2019; Accepted for publication: 24 January 2020; Published online: 11 February 2020

Keywords: diagnosis • GeneXpert • I-PCR • IGRAs • LAMP • PCR/M-PCR • UGTB

Urogenital tuberculosis (UGTB) is the second most common form of extrapulmonary TB (EPTB) in developing nations with severe epidemic situations. For example, India (comprising $45 \%$ of EPTB cases) and the third most common form in locations with a lower incidence of TB [1,2], which include both renal and genital TB. However, concomitant UGTB and pulmonary TB (PTB) are found in $2-10$ and $15-20 \%$ of patients in developed and developing nations, respectively [2]. In fact, UGTB occurs after a prolonged period of latent infection followed by hematogenous spread to the kidneys, fallopian tubes and epididymis [3]. The clinical manifestations of female UGTB include primary or secondary infertility, menstrual dysfunction and pelvic inflammatory disease [4]. Infertility is the most common complication, which occurs in $5-16 \%$ of Indian women caused by irreversible damage to the fallopian tubes. Mostly, fallopian tubes are associated with congestion and miliary tubercles, while endometrium is linked with caseation and ulceration in 50-80\% cases, resulting in Asherman's syndrome [5]. Similarly, the most frequent sites for male UGTB are the epididymis and prostate followed by seminal vesicles and testicles originating from renal foci, which also lead to infertility [6]. Owing to asymptomatic nature and varied clinical presentations (ranging from vague urinary symptoms to chronic kidney disease) that mimic several urologic and genital diseases, diagnosis of UGTB is a daunting challenge [3].

Smear/culture, histopathology, interferon- $\gamma$ release assays (IGRAs), intravenous pyelography, laparoscopy and nucleic acid amplification tests (NAATs) are the main modalities employed in the diagnosis of UGTB [6-8], which have certain limitations. Smear microscopy and culture for Mycobacterium tuberculosis identification (on Lowenstein-Jensen medium or BACTEC-460/MGIT-960) are commonly used methods but they lead to poor sensitivities due to paucibacillary nature of specimens [7]. Though urine culture is considered the gold standard, it requires skillful technicians and has a prolonged turnaround time of approximately 6-8 weeks [7] when irreparable damage to the fallopian tubes and epididymis already occurs. Delayed diagnosis also leads to nonfunctioning unilateral kidney, contracted bladder and renal failure [1]. Endoscopic procedures such as laparoscopy/hysteroscopy are often linked with operative risks and mostly cause flaring of infection [5], while histopathological examination can be confirmatory only when it demonstrates acid-fast bacilli in the tissues that are very scarce in endometrial biopsies (EBs) [7]. Intravenous pyelography may be suggestive of UGTB but it is nonspecific and often mimics other pathologic lesions [1].

\section{IGRAs}

Immunological procedures such as IGRAs are widely employed for the early detection of UGTB cases. Notably, Kim et al. [8] reported a sensitivity of $52.6 \%$ by Quantiferon-TB Gold in-Tube assay with the peripheral blood of both male and female UGTB patients using ESAT-6 (Rv3875), CFP-10 (Rv3874) and TB 7.7 as eliciting agents and proposed this test to be a supplementary tool along with urine smear/culture. On the other hand, enzyme-linked immunospot assay for interferon- $\gamma$ production revealed a higher sensitivity of $86.4 \%$ but a lesser

Future $\because \cdots$ Medicine 
specificity (75.4\%) in female UGTB patients [9]. Moreover, T-SPOT.TB test with pelvic effusion exhibited better sensitivity than peripheral blood.

\section{NAATS}

Molecular tests such as NAATs, in other words, loop-mediated isothermal amplification (LAMP), PCR, multiplexPCR (M-PCR), real-time PCR and GeneXpert (MTB/RIF) are quite popular these days for the rapid diagnosis of EPTB cases, including UGTB.

LAMP: Though LAMP test has been well-documented for the diagnosis of PTB, TB meningitis and intraocular TB [7], there is only one report to detect UGTB cases. For example, Sethi et al. [7] designed LAMP targeting IS6110 using EBs of female UGTB that exhibited moderate sensitivity and specificity though male UGTB specimens were not exploited. To further enhance the accuracy, we are currently focused to design LAMP test using multiplegene target approach with 2 or 3 targets from a pool of genes, in other words, IS6110, mpt64 (Rv1980c), fbp (Rv1886c), esat6 - among others, within urine samples of both men and women. Indeed, LAMP has the potential to revolutionize UGTB diagnosis especially in resource-limited settings as it utilizes a simple dry water-bath for amplification and avoids the use of a thermocycler, which may translate into a simple, reliable and cost-effective point-of-care test.

PCR: PCR tests targeting IS6110, hsp65 (Rv0440), mpt64, fbp, mtp40 (Rv2351c), TRC4 (conserved repetitive element) - among others, have been devised using endometrial aspirates, EBs, renal biopsies, menstrual blood and urine specimens $[2,4,10]$ but they often lead to false-positive and false-negative results. Detection of male UGTB within semen samples by PCR has also been reported that revealed superiority over MGIT-960 culture [6]. However, Radhika et al. [11] improved the sensitivity of monoplex nested-PCR targeting IS 6110 by combining with BACTEC-460 culture in diagnosing female UGTB. In a recent meta-analysis of eight studies conducted with the urine samples of UGTB patients from the diverse geographical regions by PCR [2], heterogeneous sensitivities were observed, which could be due to different gene targets employed.

M-PCR/real-time PCR: To improve the sensitivity of PCR, Bhanothu et al. [10] designed M-PCR using $m p t 64+L p q H(\mathrm{Rv} 3763)+T R C 4+m p t 59-\alpha(f b p)$ within EBs, ovarian biopsies and pelvic aspirates of suspected female UGTB cases. Compared with smear/culture and histopathology, M-PCR showed an improvement for the detection of UGTB cases. Since the collection of menstrual blood is a noninvasive procedure, M-PCR targeting mpt64+IS6110 has been devised within menstrual blood specimens and compared results with EBs [12], where menstrual blood revealed better sensitivity than EBs. Similarly, a comparison was made between the menstrual blood and EB specimens by M-PCR using IS6110+dnaJ (Rv0352)+hsp65 targets [13], which showed lesser sensitivity but higher specificity with menstrual blood, while the reverse was true with EBs. The utility of M-PCR targeting hsp65+esat6+its (16S-23S internal transcribed spacer sequence) for differential detection of M. tuberculosis and $M$. avium complex from the suspected female UGTB cases has been documented, which exhibited better sensitivity than laparoscopy/hysteroscopy [4]. Interestingly, diagnosis of renal TB from renal biopsy specimens by real-time PCR targeting IS6110 was demonstrated using 35- and 40-cycle threshold (CT) cut-off values, which revealed higher sensitivity but lesser specificity with CT-40, while the reverse was noted with CT-35 [14]. Taken together, positive M-PCR results along with clinical findings is a good choice to start antitubercular therapy in suspected UGTB cases, when the other conventional tests almost fail.

GeneXpert: GeneXpert assay, a semi-nested real-time PCR using rpoB (Rv0664) has been a major breakthrough during the last decade for the identification of both $M$. tuberculosis and rifampin resistance [15,16] though limited information is available to detect UGTB specimens by this assay. While analyzing premenstrual EBs for diagnosing 240 infertile women, Xpert revealed a low sensitivity (46.6\%) but a high specificity of $100 \%$ [16]. In another study [15], a moderate sensitivity of $63 \%$ but a high specificity $(98.8 \%)$ was attained by Xpert within urine samples of male and female UGTB patients. These findings indicate that Xpert can be used as an excellent rule-in test but not as rule-out test. Moreover, wide utilization of this test in developing countries like India is restricted primarily due to high cost.

\section{Immuno-PCR}

Notably, NAATs are restricted to detect nucleic acid molecules only; however, there are abundant protein/lipid molecules in circulation during UGTB infection, which remain unexplored. Although ELISA can detect nonnucleic acid molecules, it fails when the target antigen concentration is low. Contrarily, Immuno-PCR (I-PCR) assay can detect ultralow amount of target antigens [17]. In fact, I-PCR comprises the versatility of ELISA with 
the enormous amplification capacity of PCR, thereby exhibiting many-fold higher sensitivity than ELISA [17]. Moreover, urine is quite easy to obtain that has a lesser risk of infection to the laboratory workers but it carries low concentration of $M$. tuberculosis biomarkers [18]. Detection of mycobacterial lipoarabinomannan (LAM) within urine samples is considered as an attractive biomarker, which has been demonstrated in HIV-coinfected and HIVuninfected TB individuals by lateral flow immunochromatography and ELISA methods though poor sensitivities were observed in HIV-uninfected TB individuals [17,19]. However, urinary extracellular vesicles (EVs) isolated from TB patients can furnish intact pathogen-derived biomarkers and also concentrate those biomarkers [19]. We recently detected CFP-10 and LAM from the urinary EVs of PTB and EPTB patients by I-PCR, which revealed significantly higher sensitivities than ELISA [19]. Furthermore, sensitivities attained with LAM detection by I-PCR were better than CFP-10 detection, but urinary EVs of UGTB patients were not examined. To further improve the diagnosis of UGTB, we recommend detection of a cocktail of $M$. tuberculosis biomarkers, for example, LAM+CFP-10/MPT64 within urinary EVs of UGTB patients by I-PCR/nanoparticle-based I-PCR assay, which may match the WHO criteria of high-priority target product profile [19].

\section{Circulating cell-free DNA}

Identification of circulating cell-free DNA by PCR/real-time PCR in bodily fluids, for example, plasma, urine and pleural fluids of TB patients has recently been documented [20]. The prospective of circulating cell-free DNA as a noninvasive diagnostic method seems to be encouraging for UGTB as it may allow the diagnosis of disease from easily available blood/urine samples and that can develop into a point-of-care test.

In brief, the efficient diagnosis of UGTB by reliable tests would certainly reduce the infertility in both men and women and the other sequelae associated with disease.

\section{Financial \& competing interests disclosure}

The financial support provided by the Indian Council of Medical Research, (5/8/5/38/2014-ECD-I), New Delhi, India is being acknowledged. PKM acknowledges the Council of Scientific and Industrial Research (CSIR), New Delhi, India for awarding the Emeritus Scientist fellowship to pursue this study. EK also acknowledges CSIR for awarding Junior Research Fellowship. The authors have no other relevant affiliations or financial involvement with any organization or entity with a financial interest in or financial conflict with the subject matter or materials discussed in the manuscript apart from those disclosed.

No writing assistance was utilized in the production of this manuscript.

\section{References}

1. Kulchavenya E, Kholtobin D. Diseases masking and delaying the diagnosis of urogenital tuberculosis. Ther. Adv. Urol. 7(6), 331-338 (2015).

2. Altez-Fernandez C, Ortiz V, Mirzazadeh M, Zegarra L, Seas C, Ugarte-Gil C. Diagnostic accuracy of nucleic acid amplification tests (NAATs) in urine for genitourinary tuberculosis: a systematic review and meta-analysis. BMC Infect. Dis. 17(1), 390 (2017).

3. Kim EJ, Lee W, Jeong WY et al. Chronic kidney disease with genitourinary tuberculosis: old disease but ongoing complication. BMC Nephrol. 19(1), 193 (2018).

4. Sankar MM, Kumar P, Munawwar A et al. Usefulness of multiplex PCR in the diagnosis of genital tuberculosis in females with infertility. Eur. J. Clin. Microbiol. Infect. Dis. 32(3), 399-405 (2013).

5. Baradwan S, Shafi D, Baradwan A et al. The effect of endometrial thickness on pregnancy outcome in patients with Asherman's syndrome post-hysteroscopic adhesiolysis. Int. J. Womens Health 10, 77-82 (2018).

6. Regmi SK, Singh UB, Sharma JB, Kumar R. Relevance of semen polymerase chain reaction positive for tuberculosis in asymptomatic men undergoing infertility evaluation. J. Hum. Reprod. Sci. 8(3), 165-169 (2015).

7. Sethi S, Dhaliwal L, Dey P, Kaur H, Yadav R. Loop-mediated isothermal amplification assay for detection of Mycobacterium tuberculosis complex in infertile women. Indian J. Med. Microbiol. 34(3), 322-327 (2016).

8. Kim JK, Bang WJ, Oh CY, Yoo C, Cho JS. Feasibility of the interferon- $\gamma$ release assay for the diagnosis of genitourinary tuberculosis in an endemic area. Korean J. Urol. 54(2), 123-126 (2013).

9. $\mathrm{Lu} \mathrm{X}, \mathrm{Li}$ C, Li W et al. Use of interferon- $\gamma$ release assay for the diagnosis of female genital tuberculosis in Northwest China. J. Clin. Lab. Anal. 33(1), e22621 (2019).

10. Bhanothu V, Theophilus JP, Rozati R. Use of endo-ovarian tissue biopsy and pelvic aspirated fluid for the diagnosis of female genital tuberculosis by conventional versus molecular methods. PLoS ONE 9(5), e98005 (2014).

11. Radhika AG, Bhaskaran S, Saran N, Gupta S, Radhakrishnan G. Comparison of diagnostic accuracy of PCR and BACTEC with Lowenstein-Jensen culture and histopathology in the diagnosis of female genital tuberculosis in three subsets of gynaecological conditions. J. Obstet. Gynaecol. 36(7), 940-945 (2016). 
12. Ghosh SK, Mondal R. Quick diagnosis of female genital tuberculosis using multiplex fast polymerase chain reaction in Southern Assam, India. Int. J. Gynecol. Obstet. 118(1), 72-73 (2012).

13. Paine SK, Basu A, Choudhury RG, Bhattacharya B, Chatterjee S, Bhattacharya C. Multiplex PCR from menstrual blood: a non-invasive cost-effective approach to reduce diagnostic dilemma for genital tuberculosis. Mol. Diagn. Ther. 22(3), 391-396 (2018).

14. Kumar P, Singh S, Usha et al. Diagnosis of renal tuberculosis by real-time polymerase chain reaction in renal biopsy sample. W.J.P.M.R 3(8), 285-288 (2017).

15. Pang Y, Shang Y, Lu J et al. GeneXpert MTB/RIF assay in the diagnosis of urinary tuberculosis from urine specimens. Sci. Rep. 7(1), 6181 (2017).

16. Sharma JB, Kriplani A, Dharmendra S, Chaubey J, Kumar S, Sharma SK. Role of GeneXpert in diagnosis of female genital tuberculosis: a preliminary report. Eur. J. Obstet. Gynecol. Reprod. Biol. 207, 237-238 (2016).

17. Mehta PK, Dahiya B, Sharma $S$ et al. Immuno-PCR, a new technique for the serodiagnosis of tuberculosis. J. Microbiol. Methods 139, 218-229 (2017).

18. Turbawaty DK, Sugianli AK, Soeroto AY, Setiabudiawan B, Parwati I. Comparison of the performance of urinary Mycobacterium tuberculosis antigens cocktail (ESAT6, CFP10, and MPT64) with culture and microscopy in pulmonary tuberculosis patients. Int. J. Microbiol. 2017, 3259329 (2017).

19. Dahiya B, Khan A, Mor P et al. Detection of Mycobacterium tuberculosis lipoarabinomannan and CFP-10 (Rv3874) from urinary extracellular vesicles of tuberculosis patients by immuno-PCR. Pathog. Dis. 77(5), ftz049 (2019).

20. Fernández-Carballo BL, Broger T, Wyss R, Banaei N, Denkinger CM. Toward the development of a circulating free DNA-based in vitro diagnostic test for infectious diseases: a review of evidence for tuberculosis. J. Clin. Microbiol. 57(4), e01234-18 (2019). 